\title{
Slow dynamics at the smeared phase transition of randomly layered magnets
}

\author{
Shellie Huether, Ryan Kinney, and Thomas Vojta \\ Department of Physics, University of Missouri - Rolla, Rolla, MO 65409, USA
}

(Dated: November 20, 2018)

\begin{abstract}
We investigate a model for randomly layered magnets, viz. a three-dimensional Ising model with planar defects. The magnetic phase transition in this system is smeared because static long-range order can develop on isolated rare spatial regions. Here, we report large-scale kinetic Monte Carlo simulations of the dynamical behavior close to the smeared phase transition which we characterize by the spin (time) autocorrelation function. In the paramagnetic phase, its behavior is dominated by Griffiths effects similar to those in magnets with point defects. In the tail region of the smeared transition the dynamics is even slower: the autocorrelation function decays like a stretched exponential at intermediate times before approaching the exponentially small asymptotic value following a power law at late times. Our Monte-Carlo results are in good agreement with recent theoretical predictions based on optimal fluctuation theory.

PACS numbers: 64.60.Ht, 05.50.+q, 75.10.Nr, 75.40.Gb
\end{abstract}

\section{INTRODUCTION}

In recent years, there has been a resurgent interest in the influence of defects, impurities or other types of quenched disorder on the properties of phase transitions and critical phenomena. This renewed attention is largely due to the discoveries of novel disorder effects that go beyond the framework of perturbation theory and the perturbative renormalization group.

A particularly interesting class of non-perturbative phenomena are the so-called Griffiths or rare region effects that are produced by rare strong spatial disorder fluctuations. They can be easily understood on the example of a diluted magnet: Due to the dilution, the critical temperature $T_{c}$ of the disordered system is lower than its clean value, $T_{c}^{0}$. In the temperature interval $T_{c}<T<T_{c}^{0}$, the diluted bulk system is in the disordered phase. However, in an infinite size sample, there is an exponentially small, but nonzero probability for finding an arbitrary large spatial region devoid of impurities. Such a rare region, a 'Griffiths island', displays local order in the temperature interval $T_{c}<T<T_{c}^{0}$. Due to its size, such an island will have very slow dynamics because flipping it requires changing the order parameter over a large volume. More than 30 years ago, Griffiths ${ }^{1}$ showed that the presence of these locally ordered islands produces a singularity in the free energy in the whole region $T_{c}<T<T_{c}^{0}$, which is now known as the Griffiths region or the Griffiths phase. ${ }^{2}$ In generic classical systems with uncorrelated or short-range correlated disorder, thermodynamic Griffiths effects are very weak because the singularity in the free energy is only an essential one. ${ }^{3-5}$ To the best of our knowledge, classical thermodynamic Griffiths singularities have therefore not been verified in experiment (see also Ref. 6).

In contrast to the thermodynamics, the long-time $d y$ namics is dominated by the rare regions. Inside the Griffiths phase, the spin autocorrelation function $C(t)$ decays very slowly with time $t$, as $\ln C(t) \sim-(\ln t)^{d /(d-1)}$ for Ising systems ${ }^{2,7-10}$ and as $\ln C(t) \sim-t^{1 / 2}$ for Heisen- berg systems. ${ }^{9,11}$ These results were recently confirmed by more rigorous calculation for the equilibrium ${ }^{12,13}$ and dynamic $^{14,15}$ properties of disordered Ising systems.

The effects of impurities and defects are greatly enhanced by long-range spatial disorder correlations. In particular, if the disorder is perfectly correlated in some spatial directions, the rare regions are extended objects which are infinite in the correlated dimensions. This makes their dynamics even slower and so increases their effects. In Ising models with linear defects (disorder perfectly correlated in one dimension), the thermodynamic Griffiths singularities are of power-law type, with the average susceptibility actually diverging in a finite temperature region. The critical point itself is an exotic infinite-randomness critical point and displays activated rather than power-law scaling. This was first found in the McCoy-Wu model, ${ }^{16,17}$ a disordered 2D Ising model in which the disorder is perfectly correlated in one dimension and uncorrelated in the other. Later it was studied in great detail in the context of the quantum phase transition of the random transverse-field Ising model where the imaginary time dimension plays the role of the "correlated" direction. ${ }^{18,19}$

Recently, it has been shown that even stronger effects than these power-law Griffiths singularities can occur in Ising models with planar defects. ${ }^{20,21}$ Because the disorder is perfectly correlated in two directions, the effective dimensionality of the rare regions is two. Therefore, an isolated rare region can undergo the phase transition independently from the bulk system. This leads to a destruction of the global sharp phase transition by smearing. Similar smearing effects have also been found in itinerant quantum magnets ${ }^{22}$ and at a non-equilibrium transition in the presence of linear defects ${ }^{23}$. A recent review of these and other rare region effects can be found in Ref. 24.

In this paper, we study the dynamics of an Ising model with planar defects in the vicinity of this smeared phase transition by large-scale kinetic Monte-Carlo simulations. The paper is organized as follows. In Section II we in- 
troduce the model and briefly summarize the results of the optimal fluctuation theory ${ }^{20,24,25}$ for smeared phase transitions to the extent necessary for the analysis of our data. In section III, we explain the simulation technique, we present our results for the spin autocorrelation function, and we compare them to the theoretical predictions. Conclusions are presented in section IV.

\section{THEORY}

\section{A. Three-dimensional Ising model with planar defects}

We consider a three-dimensional classical Ising ferromagnet with planar defects, the same model whose thermodynamics was studied in Ref. 21. Ising variables $S_{i, j, k}= \pm 1$ reside on the sites of a cubic lattice. They interact via nearest-neighbor interactions. In the clean system all interactions are identical and have the value $J$. The defects are modeled via 'weak' bonds randomly distributed in one dimension (uncorrelated direction). The bonds in the remaining two dimensions (correlated directions) remain equal to $J$. The system effectively consists of strongly-coupled slabs (layers) of varying thickness, separated by parallel layers of weak bonds. The Hamiltonian of the system is given by:

$$
\begin{aligned}
H= & -\sum_{\substack{i=1, \ldots, L_{\perp} \\
j, k=1, \ldots, L_{C}}} J_{i} S_{i, j, k} S_{i+1, j, k} \\
& -\sum_{\substack{i=1, \ldots, L_{\perp} \\
j, k=1, \ldots, L_{C}}} J\left(S_{i, j, k} S_{i, j+1, k}+S_{i, j, k} S_{i, j, k+1}\right),(1)
\end{aligned}
$$

where $L_{\perp}\left(L_{C}\right)$ is the length in the uncorrelated (correlated) direction, $i, j$ and $k$ are integers counting the sites of the cubic lattice, $J$ is the interaction energy in the correlated directions and $J_{i}$ is the random interaction in the uncorrelated direction. The $J_{i}$ are drawn from a binary distribution:

$$
J_{i}=\left\{\begin{array}{cl}
c J & \text { with probability } p \\
J & \text { with probability } 1-p
\end{array}\right.
$$

characterized by the concentration $p$ with $0 \leq p<1$ and the relative strength $c$ of the weak bonds $(0<c \leq 1)$.

The Hamiltonian (1) does not contain any internal dynamics. Instead, the dynamics must be added ad-hoc by specifying an appropriate dynamical algorithm. We consider a purely relaxational local dynamics without any conservation laws, i.e., model $\mathrm{A}$ in the HohenbergHalperin classification. ${ }^{26}$ Microscopically, it can be realized, e.g., by the Glauber or Metropolis algorithms. ${ }^{27,28}$

\section{B. Smeared phase transition and optimal fluctuation theory}

In the absence of impurities $(p=0)$, the system (1) undergoes a ferromagnetic phase transition at the clean critical temperature $T_{c}^{0}=4.511 \mathrm{~J}$, with the order parameter being the total magnetization:

$$
m=\frac{1}{V} \sum_{i, j, k}\left\langle S_{i, j, k}\right\rangle
$$

Here, $V=L_{\perp} L_{C}^{2}$ is the volume of the system, and $\langle\cdot\rangle$ denotes the thermodynamic average.

In the presence of disorder $(p>0)$, a crucial role is played by rare strong disorder fluctuations: In analogy to the Griffiths phenomena ${ }^{1}$ discussed in the Introduction, there is a small but finite probability for finding a large spatial region containing only strong bonds in the uncorrelated direction. Such a rare region can be locally in the ferromagnetic state while the bulk system is still in the disordered (paramagnetic) phase. The ferromagnetic order on the largest rare regions starts to emerge right below the clean critical temperature $T_{c}^{0}$. Since the defects in the system are planar, these rare regions are infinite in the two correlated dimensions but finite in the uncorrelated direction. Each rare region is thus equivalent to a two-dimensional Ising system that can undergo a real phase transition independently of the rest of the system. The resulting effect is much stronger than conventional Griffiths effects: the global phase transition is destroyed by smearing, and the order parameter develops very inhomogeneously in space with different parts of the system (different rare regions) ordering independently at different temperatures..$^{20,21,24}$

The leading thermodynamic behavior in the tail of the smeared transition can be determined using optimal fluctuation theory. ${ }^{20,24}$ The approach is similar to that of Lifshitz ${ }^{29}$ and others for the description of the tails in the electronic density of states of disordered systems. The theory can be easily developed for a general $d$-dimensional system with $d_{C}$ correlated dimensions and $d_{\perp}=d-d_{C}$ uncorrelated dimensions. In the case of the Hamiltonian (1), $d_{C}=2$ and $d_{\perp}=1$.

In the tail region of the smeared transition, the system consists of a few isolated ferromagnetic rare regions embedded in a paramagnetic bulk. We start from the probability $w$ for finding a large region of linear size $L_{R R}$ containing only strong bonds. Up to pre-exponential factors, it is given by

$$
w \sim(1-p)^{L_{R R}^{d_{\perp}}}=\exp \left[L_{R R}^{d_{\perp}} \ln (1-p)\right] .
$$

Such a rare region develops static ferromagnetic longrange order at some temperature $T_{c}\left(L_{R R}\right)$ below the clean critical temperature $T_{c}^{0}$. The value of $T_{c}\left(L_{R R}\right)$ varies with the size of the rare region; the largest islands will develop long-rage order closest to the clean critical point. To determine $T_{c}\left(L_{R R}\right)$, we can use finite size scaling ${ }^{30}$ for the clean system because a rare region is equivalent to a "slab" of a clean Ising model. This yields

$$
T_{c}^{0}-T_{c}\left(L_{R R}\right)=\left|r_{c}\left(L_{R R}\right)\right|=A L_{R R}^{-\phi},
$$


where $\phi$ is the finite-size scaling shift exponent of the clean system and $\mathrm{A}$ is the amplitude for the crossover from $d=d_{C}+d_{\perp}$ dimensions to a slab geometry infinite in $d_{C}$ (correlated) dimension but with finite length in the other (uncorrelated) directions. The reduced temperature $r=T-T_{c}^{0}$ measures the distance from the clean critical point. As long as the clean $d$-dimensional Ising model is below its upper critical dimension $\left(d_{c}^{+}=4\right)$, hyperscaling is valid and the finite-size shift exponent is related to the correlation length exponent $\nu$ by $\phi=1 / \nu$ which we assume from now on. Combining (4) and (5), we obtain the probability for finding an island which becomes critical at some $r_{c}$ as:

$$
w\left(r_{c}\right) \sim \exp \left(-B\left|r_{c}\right|^{-d_{\perp} \nu}\right) \quad\left(\text { for } r_{c} \rightarrow 0-\right)
$$

with the constant $B=-\ln (1-p) A^{d_{\perp} \nu}$. The total (average) magnetization $m$ at some reduced temperature $r$ is obtained by integrating over all rare regions which are ordered at $r$, i.e., those with $r_{c}>r$. Since the functional dependence on $r$ of the local magnetization on the island is of power-law type it does not enter the leading exponentials but only pre-exponential factors. To exponential accuracy, we therefore obtain

$$
m(r) \sim \exp \left(-B|r|^{-d_{\perp} \nu}\right) \quad(\text { for } r \rightarrow 0-) .
$$

Thus, the total magnetization develops an exponential tail towards the disordered phase which reaches all the way to the clean critical point. Analogous estimates show that the homogeneous magnetic susceptibility does not diverge anywhere in the tail region of the smeared transition. At $r=0$, the exponentially decreasing island density overcomes the power-law divergence of the susceptibility of an individual island; and once a nonzero magnetization has developed, it cuts off any possible singularity. However, there is an essential singularity in the free energy at the clean critical point produced by the vanishing density of ordered islands.

\section{Dynamics at the smeared transition}

After having briefly discussed the optimal fluctuation theory for the thermodynamics, we now consider the dynamical behavior at the smeared phase transition of our disordered magnet (1). The interesting physics in the tail of the smeared transition is local with respect to the uncorrelated dimensions because different rare regions are effectively decoupled from each other, and the spatial correlation length in these directions remains finite. An appropriate quantity to study the rare region dynamics is therefore the time autocorrelation function of the Ising spins,

$$
C(t)=\frac{1}{V} \sum_{i, j, k}\left\langle S_{i, j, k}(t) S_{i, j, k}(0)\right\rangle .
$$

The leading long-time behavior of $C(t)$ in the tail of the smeared transition can be determined using optimal fluctuation arguments similar to that of section II B. ${ }^{24,25}$
According to finite-size scaling ${ }^{30}$, the behavior of the correlation time $\xi_{t}$ of a single rare region of size $L_{R R}$ in the vicinity of the clean bulk critical point can be modelled by (for $T<T_{c}^{0}$, i.e., $r<0$ )

$$
\xi_{t}\left(r, L_{R R}\right) \sim L_{R R}^{(z \nu-\tilde{z} \tilde{\nu}) / \nu}\left|r+A L_{R R}^{-1 / \nu}\right|^{-\tilde{z} \tilde{\nu}} .
$$

Here, $\nu$ and $z$ are the correlation length and dynamical exponents of a $d$-dimensional system, and $\tilde{\nu}$ and $\tilde{z}$ are the corresponding exponents of a $d_{C}$-dimensional system.

Let us first consider the time evolution of the autocorrelation function $C(t)$ at the clean critical point $T_{c}^{0}$, i.e., the boundary between the conventional paramagnetic phase and the smeared tail of the ordered phase. For $r=0$, the correlation time (9) simplifies to $\xi_{t} \sim L_{R R}^{z}$. The rare region contribution to $C(t)$ is obtained by simply summing over the exponential time dependencies of the individual islands with $L_{R R^{-}}$dependent correlation time. Using (4) we obtain to exponential accuracy

$$
C(t) \sim \int d L_{R R} \exp \left(L_{R R}^{d_{\perp}} \ln (1-p)-D t / L_{R R}^{z}\right)
$$

where $D$ is a constant. This integral can be easily estimated within the saddle-point method. The leading long-time decay of the autocorrelation function $C(t)$ at the clean critical point is given by a stretched exponential,

$$
\ln C(t) \sim-[-\ln (1-p)]^{z /\left(d_{\perp}+z\right)} t^{d_{\perp} /\left(d_{\perp}+z\right)} \quad(r=0) .
$$

In the conventional paramagnetic phase, $T>T_{c}^{0}$ $(r>0)$, the correlation time does not diverge for any $L_{R R}$. Instead, the correlation time of the large islands saturates at $\xi_{t}\left(r, L_{R R}\right) \sim r^{-z \nu}$ for $L_{R R}>\left(r / A^{\prime}\right)^{-\nu}$. The autocorrelation function $C(t)$ can again be evaluated as an integral over all island contributions. We find that there are two regimes separated by a crossover time

$$
t_{x} \sim|r|^{-\left(d_{\perp}+z\right) \nu} .
$$

For intermediate times $t<t_{x}$, the autocorrelation function follows the stretched exponential (11). For times larger than the crossover time $t_{x}$, we obtain a simple exponential decay

$$
\ln C(t) \sim-t / \tau \quad\left(\text { for } t>t_{x}, r>0\right)
$$

with the decay time $\tau \sim r^{-z \nu}$. Our results for $T \geq T_{c}^{0}$ agree with the corresponding conventional dynamical Griffiths effects ${ }^{9}$ for systems with point defects. This is not surprising, because above $T_{c}^{0}$, there is no qualitative difference between the Griffiths and the smearing scenarios: In both cases, all rare regions are locally still in the disordered phase.

This changes in the tail of the smeared transition below the clean critical point $T_{c}^{0}$. For $r<0$, we repeat the saddle point analysis with the full expression (9) for the correlation length. Again, for intermediate times $t<t_{x}$, 
the decay of the average density is given by the stretched exponential (11). For times larger than the crossover time $t_{x}$ the system realizes that some of the rare regions have developed static order and contribute to a non-zero long-time limit of the autocorrelation function $C(t)$. The approach of $C(t)$ to this limiting value is dominated by finite-size islands with $L_{R R} \sim(-r / A)^{-\nu}$ because they have diverging correlation time. As a result, we obtain a power-law,

$$
C(t)-C(\infty) \sim t^{-\psi} \quad\left(\text { for } t>t_{x}, r<0\right) .
$$

The value of $\psi$ cannot be found by our methods since it depends on the neglected pre-exponential factors.

In Ref. 25, the predictions (11), (13), and (14) have been compared to results from a local dynamical meanfield theory. The purpose of the present paper is to verify these relations by large-scale kinetic Monte-Carlo simulations of a realistic model with short-range interactions, viz. the Hamiltonian (1).

\section{COMPUTER SIMULATIONS}

\section{A. Method}

We now turn to the main part of the paper, kinetic Monte-Carlo simulations of our three-dimensional Ising model with nearest neighbor interactions and planar bond defects, as given in eq. (1). Since the smearing of the transition is a result of exponentially rare events, sufficiently large system sizes are required in order to observe it. In the production runs, we have mostly used system sizes of $260^{3}$ or $300^{3}$ sites. In this context, let us briefly comment on finite-size effects. Because the phase transition in an Ising model with planar defects is smeared, conventional finite-size scaling ${ }^{30}$ does not directly apply. Nonetheless, there are two types of finitesize effects, whose consequences for the thermodynamics were discussed in detail in Refs. 20,21. A finite size in the uncorrelated direction limits the possible sizes of the rare regions and thus cuts off the probability distribution (6), while a finite size in the correlated directions rounds the local phase transitions of the rare regions. We have carried out test runs using $50^{3}, 100^{3}$ and $200^{3}$ sites to ensure that our results are not influenced by these finitesize effects.

We have chosen $J=1$ and $c=0.1$ in eq. (2), i.e., the strength of a 'weak' bond is $10 \%$ of the strength of a strong bond. The simulations have been performed for various disorder concentrations $p=\{0.2,0.3,0.4,0.5,0.6\}$ over a temperature range of $T=4.25$ to 5.00 , covering both sides of the clean critical point at $T_{c}^{0}=4.511$. To suppress the statistical disorder fluctuations we have averaged the results over a large number of disorder configurations ranging from 100 to 5000 depending on parameters.

For each disorder configuration, the simulation proceeds as follows: After the initial setup, the system

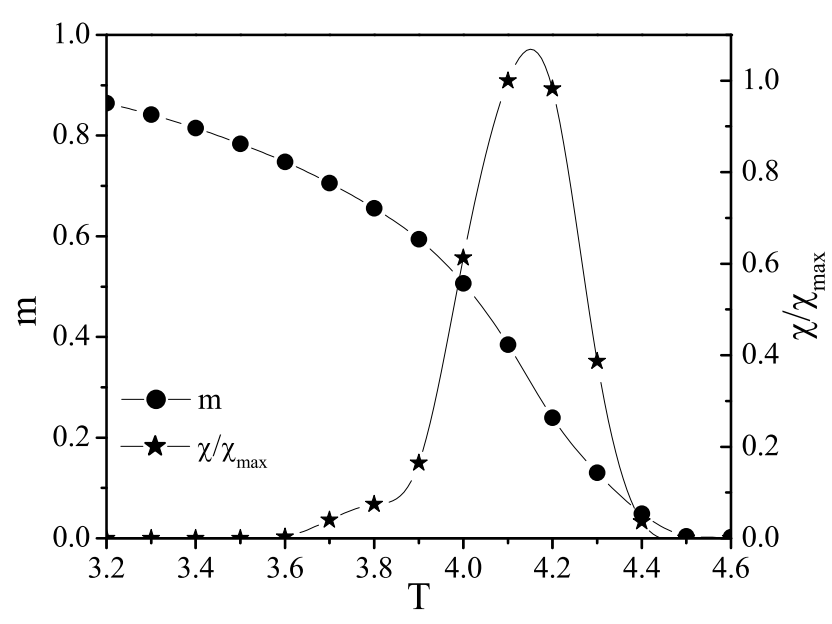

FIG. 1: Total magnetization $m$ and susceptibility $\chi$ as functions of $T$ for $L_{\perp}=100, L_{C}=200$ and $p=0.2$ averaged over 200 disorder realizations (from Ref. 21).

is equilibrated using the highly efficient Wolff cluster algorithm. ${ }^{31}$ (Using a cluster algorithm is possible for our Hamiltonian because the disorder does not induce frustration.) The length of the equilibration period is 50 Wolff sweeps. (A Wolff sweep is defined as a number of cluster flips such that the total number of flipped spins is equal to the number of sites, i.e., on the average each spin is flipped once per sweep.) The actual equilibration times have typically been of the order of 10-20 sweeps at maximum. Thus, an equilibration period of 50 sweeps is more than sufficient.

After the system is equilibrated, the spin configuration is stored and the simulation time is set to $t=0$. The actual time evolution of the system according to model A dynamics is performed using the Metropolis algorithm. ${ }^{27}$ After each sweep (a Metropolis sweep consists in one attempted spin flip per site), the spin configuration is determined and the spin autocorrelation function is calculated from (8). The length of this measurement period is up to 5000 Monte-Carlo (Metropolis) sweeps.

\section{B. Results}

In this section, we present the results of our kinetic Monte-Carlo simulations and compare them to the theoretical predictions of section II C. For later reference, we first give a brief overview of the thermodynamics, as determined in Ref. 21. Fig. 1 shows total magnetization and susceptibility as functions of temperature averaged over 200 samples of size $L_{\perp}=100$ and $L_{C}=200$ with an impurity concentration $p=0.2$. For temperatures above $T=4.2 \ldots 4.3$, the magnetization shows a pronounced tail, i.e., it vanishes very gradually when approaching the clean critical temperature $T_{c}^{0}=4.511$. It should be emphasized that this tail is not due to finite-size effects. 


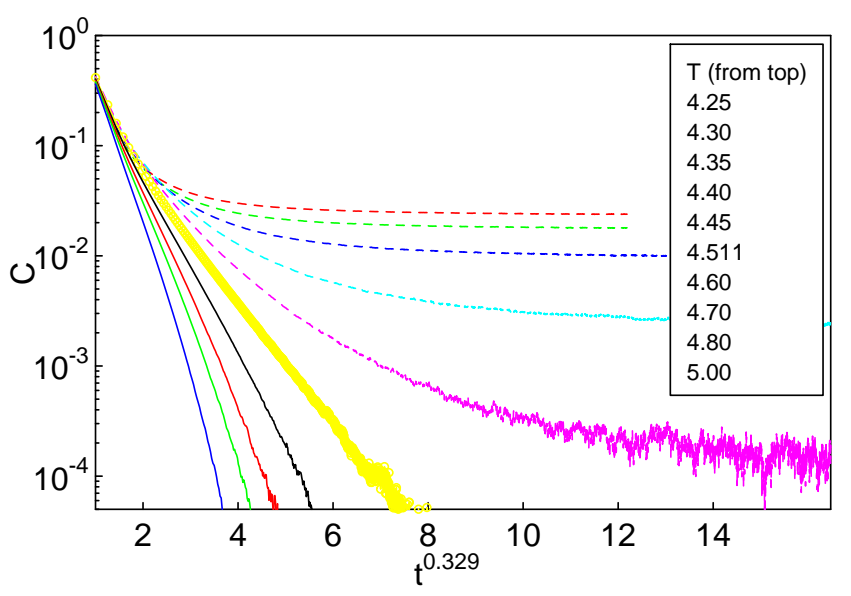

FIG. 2: Spin autocorrelation function $C$ as a function of time $t$ for $L_{\perp}=L_{C}=260, p=0.3$. The number of disorder realizations ranges from 100 at the lowest temperatures to 5000 at the highest temperatures. The power-law time axis corresponds to the stretched exponential predicted in (11).

Indeed, a detailed analysis ${ }^{21}$ has shown that the magnetization nicely follows the prediction (7) of the smeared transition scenario over more then a magnitude in $m$. Analogously, an analysis of the susceptibility has shown that it does not diverge in the thermodynamic limit, instead, it displays a rounded peak.

We now turn to the spin autocorrelation function $C(t)$. Figure 2 gives an overview over its behavior for an impurity concentration $p=0.3$ and times up to $t=5000$ over the entire temperature range studied. In this figure the data are plotted in the form $\ln C$ vs. $t^{0.329}$ such that the stretched exponential (11) predicted for the time dependence at the clean critical temperature $T_{c}^{0}=4.511$ gives a straight line. Here we have used $d_{\perp}=1$ and a value of $z=2.04$ for the dynamical exponent of the clean three-dimensional Ising model with model A dynamics (see, e.g., Refs. 32-34). The figure shows that the autocorrelation function at $T_{c}^{0}$ indeed follows the stretched exponential over more than two orders of magnitude in $C$. At temperatures $T \neq T_{c}^{0}$, the autocorrelation function initially follows the same stretched exponential but eventually crosses over to a different asymptotic form: For $T>T_{c}^{0}$, it decays faster than the stretched exponential, while it seems to saturate at a nonzero value for $T<T_{c}^{0}$. In the following, we investigate the different temperature regions in more detail.

Let us begin by analyzing the autocorrelation function $C(t)$ right at the clean critical temperature $T_{c}^{0}$. Figure 3 shows $C(t)$ for several impurity concentrations $p=0.2 \ldots 0.6$, again plotted as $\ln C$ vs. $t^{0.329}$. After the initial transients, all curves are straight lines, indicating that $C(t)$ follows the theoretical prediction (11) over at least two orders of magnitude in $C$ for all concentrations. From the curves in Fig. 3, one can determine the decay constants (i.e., the slopes) as a function of impurity concentration $p$. According to (11), the decay con-

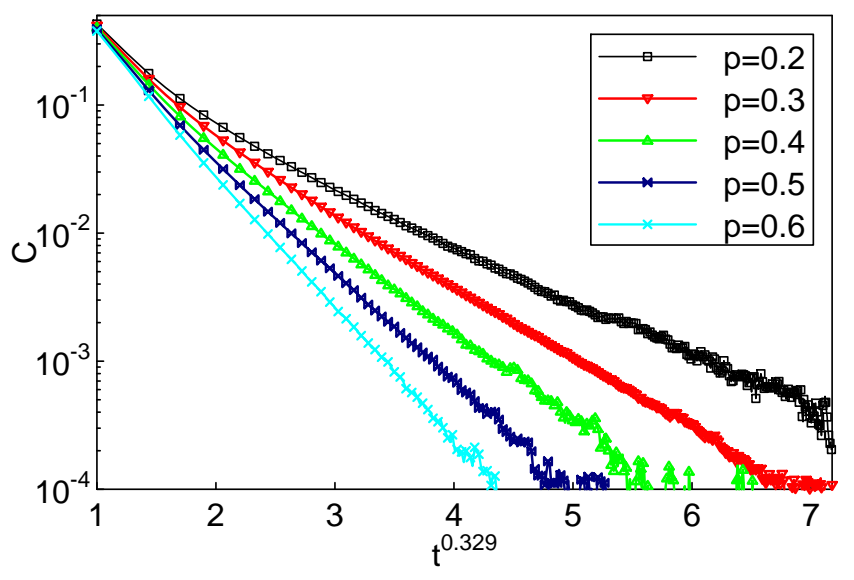

FIG. 3: Spin autocorrelation function $C(t)$ at the clean critical temperature $T_{c}^{0}=4.511$ for $L_{\perp}=L_{C}=300$ and several impurity concentrations $p$. The data are averages over 100 disorder realizations (1000 realizations for $p=0.3$ ). The statistical errors are not bigger than a symbol size for $C>10^{-3}$. For $p=0.3$, they reach about twice the symbol size at the right end of the curve.

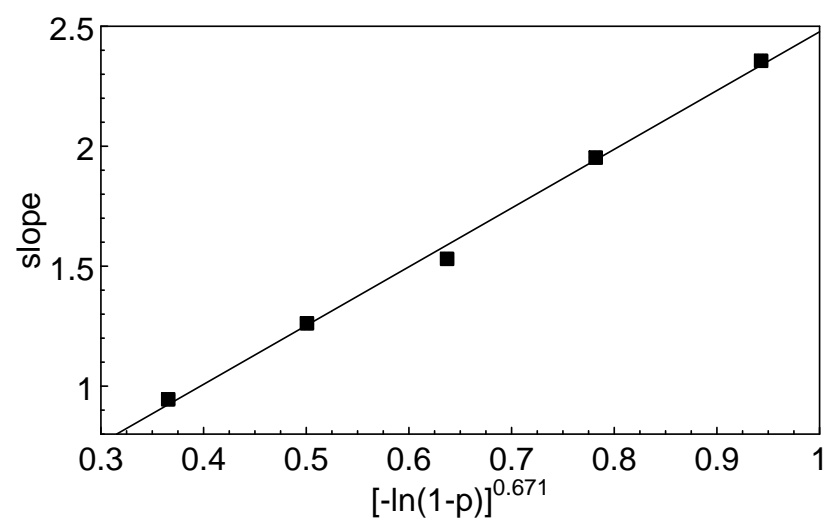

FIG. 4: Decays constants (slopes) of the stretched exponentials of Fig. 3 as a function of impurity concentration $p$, plotted according to the prediction (11).

stant should be proportional to $[-\ln (1-p)]^{z /\left(d_{\perp}+z\right)}=$ $[-\ln (1-p)]^{0.671}$. Figure 4 shows the corresponding plot for our data. It shows that the behavior of the decay constants is in good agreement with the theoretical predictions.

After the behavior at the clean critical temperature $T_{c}^{0}$, we now consider the conventional paramagnetic phase $T>T_{c}^{0}$. In Figure 5, we plot the autocorrelation function $C(t)$ for $p=0.3, L_{\perp}=L_{C}=260$, and temperatures $T>T_{c}^{0}$ as $\ln C$ vs $t$ such that a simple exponential decay gives a straight line. For all shown temperatures, the long-time behavior of the autocorrelation function is indeed an exponential decay in agreement with the theoretical prediction (13). To determine the decay time $\tau$ we fit the long-time behavior of $C(t)$ to (13). The inset of Fig. 5 shows $\tau^{-1}$ as a function of $\left(T-T_{c}^{0}\right)^{z \nu}$ with 


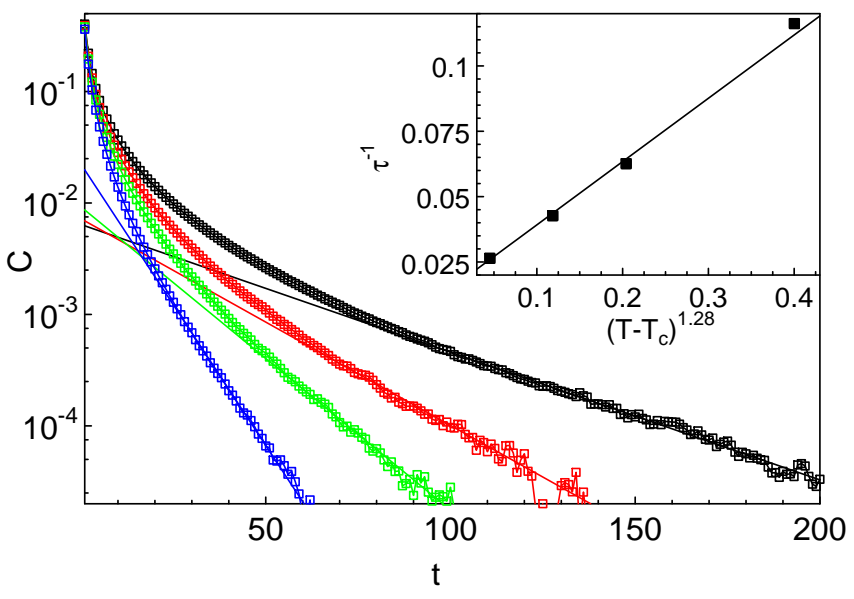

FIG. 5: Log-linear plot of the spin autocorrelation function for temperatures above $T_{c}^{0}$ (from the top: $T=4.6,4.7,4.8,5.0$ ). The other parameters are as in Fig. 2. The solid lines are fits of the long-time behavior to (13). The statistical errors are not bigger than a symbol size for $C>10^{-4}$. Inset: Inverse decay time $\tau^{-1}$ as a function of $\left(T-T_{c}^{0}\right)^{z \nu}$.

the three-dimensional clean correlation length exponent given by $\nu=0.628$ (see, e.g., Ref. 35) and $z=2.04$ as before. As predicted by the optimal fluctuation theory in Sec. II C, the inverse decay time depends linearly on $\left(T-T_{c}^{0}\right)^{z \nu}$. (The remaining small deviations can probably be attributed to the pre-exponential factors neglected in the optimal fluctuation theory.) We have performed analogous simulations for an impurity concentration of $p=0.5$ and found the same qualitative behavior. Of course, non-universal prefactors have different values.

Finally, we turn to the properties of the spin autocorrelation function $C(t)$ below the clean critical temperature $T_{c}^{0}$, i.e., in the tail of the smeared phase transition. For these temperatures, the total equilibrium magnetization is nonzero because some of the rare regions have already developed a static magnetization (see also Fig. 1). ${ }^{20,21}$ Consequently, the autocorrelation function does not decay to zero in the long-time limit but rather approaches the limiting value $m^{2}$ as can be seen in the overview figure 2. The dynamical correlations of the magnetization fluctuations $\left(S_{i, j, k}-m\right)$ are represented by the deviation $C(t)-C(\infty)$ of the autocorrelation function from its long-time limit. Figure 6 shows a double-logarithmic plot of $C(t)-C(\infty)$ for $p=0.3, L_{\perp}=L_{C}=260$, and temperatures $T=4.35,4.40$, and 4.45 . For all three temperatures, the long-time behavior of $C(t)-C(\infty)$ follows a power law. Fits to (14) give exponents of $\psi=0.80$ for $T=4.35,0.90$ for $T=4.40$, and 1.23 for $T=4.45$. Thus, as predicted, the exponent of the power-law (14) is nonuniversal. (We note that the apparent crossing of the curves in Fig. 6 is not related to the long-time dynamics but simply the result of the subtraction of $C(\infty)$. At lower temperatures, $C(\infty)$ is larger, and since $C(t)$ cannot exceed 1 , the difference $C(t)-C(\infty)$ at early times must decrease with decreasing $T$. Since in the long-time

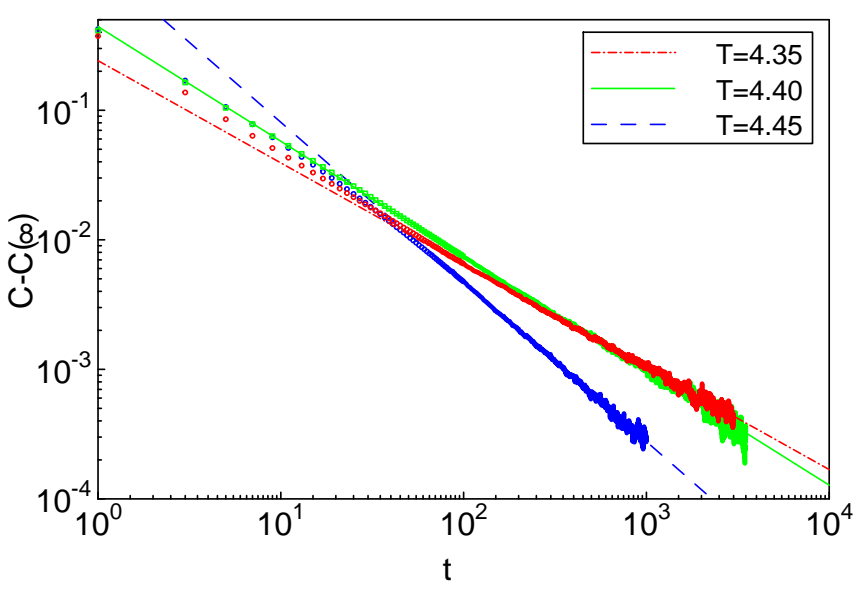

FIG. 6: Double-logarithmic plot of the dynamical part $C(t)-$ $C(\infty)$ of the autocorrelation function for temperatures below $T_{c}^{0}$. The other parameters are as in Fig. 2. The straight lines are fits of the long-time behavior to the power law (14) giving exponents of $\psi=0.80(T=4.35), 0.90(T=4.40)$ and $1.23(T=4.45)$.

limit, the decay is faster for larger $T$, the curves must cross.)

We have carried out analogous simulations for the impurity concentration $p=0.5$, and we have obtained equivalent results.

\section{CONCLUSIONS}

To summarize, we have studied the dynamic behavior of randomly layered Ising magnets by performing large-scale kinetic Monte-Carlo simulations of a threedimensional Ising model with planar defects. In this system, the magnetic phase transition is smeared because rare strongly coupled spatial regions (i.e., thick stronglycoupled layers) independently undergo the phase transition. We have found that the dynamics in the rareregion dominated tail of the smeared transition is very slow. The spin autocorrelation function approaches its stationary value following a power-law in time. At the clean critical temperature $T_{c}^{0}$ (which marks the boundary between the conventional paramagnetic phase and the tail of the smeared transition), the autocorrelation function decays like a stretched exponential in time. In the paramagnetic phase above $T_{c}^{0}$, the decay is of simple exponential type. However, the decay time $\tau$ of this exponential diverges in the limit $T \rightarrow T_{c}^{0}+$. Our numerical results (both the functional forms and the exponent values of the various time and disorder dependencies) are in very good agreement with a recent optimal fluctuation theory. ${ }^{25}$

All our explicit results are for strong impurities $(c=$ 0.1 ). We have chosen this value because strong impurities allow us to easily observe the smeared transition in a finite-size simulation. If the impurities are weak $(c$ 
close to 1), the smeared transition is too close to the clean critical point. Strong bulk fluctuations thus compete with the smearing, and the latter can only be observed in larger systems and/or at longer times. The behavior of our model for $c=0$ (dilution) is special, because for this value, the system is decomposed into an ensemble of noninteracting slabs.

We emphasize that we have considered a purely relaxational (local) dynamics corresponding to model A in the Hohenberg-Halperin classification. ${ }^{26}$ Other dynamic algorithms require separate investigations. For instance, model B dynamics globally conserves the order parameter. In this context, an interesting question is: How does the interplay of the local thermodynamics of the rare regions and the global conservation law modify the dynamic behavior in the tail of the smeared transition.

Let us briefly compare the dynamics at a smeared phase transition studied here to the behavior in a conventional Griffiths phase (as produced by uncorrelated or short-range correlated disorder such as point defects). Both in a Griffiths phase and at a smeared transition, rare regions dominate the long-time dynamics. In a conventional Griffiths phase, the finite-size rare regions remain fluctuating for all temperatures above the dirty critical point, i.e., their island correlation times remain finite. As a result, the autocorrelation function decays like $\ln C(t) \sim-(\ln t)^{d /(d-1)}$ for Ising systems ${ }^{2,7-10}$ and $\ln C(t) \sim-t^{1 / 2}$ for Heisenberg systems ${ }^{9,11}$. In contrast, in the tail of a smeared transition, the effects of the rare regions are even stronger because individual islands can undergo the phase transition independently, connected with a divergent island correlation time. Summing over all islands then leads to an even slower power-law decay of the spin autocorrelation function.

Let us also comment on experiments. A direct realization of the scenario discussed here could be achieved by growing alternating layers of two ferromagnetic materials with different critical temperatures $T_{c}$. To introduce disorder, the thickness of the layers should be random. In the tail of the smeared transition, i.e., for temperatures close to but below the higher of the two $T_{c}$, the dynamic magnetic response of this system will be dominated by the rare region contributions. Specifically, the dynamic susceptibility $\chi(\omega)$ will be dominated by its local part which is essentially the Fourier transform of the autocorrelation function. Thus measuring the dynamic response in such a randomly layered magnet should provide an experimental verification of our results.

Finally, while the results here have been derived for an Ising model with planar defects, we expect analogous results for other disorder-smeared phase transitions. Indeed, in the tail of the smeared non-equilibrium phase transition of a contact process with extended impurities, a power-law decay of the density was recently found. ${ }^{23,36}$.

\section{Acknowledgments}

We acknowledge support from the University of Missouri Research Board, from the NSF under grants No. DMR-0339147 and PHY99-07949, and from Research Corporation. Parts of this work have been performed at the Aspen Center for Physics and at the Kavli Institute for Theoretical Physics, Santa Barbara.
${ }^{1}$ R. B. Griffiths, Phys. Rev. Lett. 23, 17 (1969).

2 M. Randeria, J. P. Sethna, and R. G. Palmer, Phys. Rev. Lett. 54, 1321 (1985).

3 M. Wortis, Phys. Rev. B 10, 4665 (1974).

4 A. B. Harris, Phys. Rev. B 12, 203 (1975).

5 A. J. Bray and D. Huifang, Phys. Rev. B 40, 6980 (1989).

${ }^{6}$ Y. Imry, Phys. Rev. B 15, 4448 (1977).

7 D. Dhar, in Stochastic Processes: Formalism and Applications, edited by D. S. Argawal and S. Dattagupta (Springer, Berlin, 1983).

8 D. Dhar, M. Randeria, and J. P. Sethna, Europhys. Lett. 5, 485 (1988).

9 A. J. Bray, Phys. Rev. Lett. 60, 720 (1988).

10 A. J. Bray and G. J. Rodgers, Phys. Rev. B 38, 9252 (1988).

11 A. J. Bray, Phys. Rev. Lett. 59, 586 (1987).

12 H. v. Dreyfus, A. Klein, and J. F. Perez., Commun. Math. Phys. 170, 21 (1995).

13 G. Gielis and C. Maes, J. Stat. Phys. 81, 829 (1995).

14 F. Cesi, C. Maes, and F. Martinelli, Commun. Math. Phys. 188, 135 (1997).

15 F. Cesi, C. Maes, and F. Martinelli, Commun. Math. Phys. 189, 323 (1997).

16 B. M. McCoy and T. T. Wu, Phys. Rev. Lett. 21, 549
(1968).

17 B. M. McCoy and T. T. Wu, Phys. Rev. 176, 631 (1968).

18 D. S. Fisher, Phys. Rev. Lett. 69, 534 (1992).

19 D. S. Fisher, Phys. Rev. B 51, 6411 (1995).

20 T. Vojta, J. Phys. A 36, 10921 (2003).

21 R. Sknepnek and T. Vojta, Phys. Rev. B 69, 174410 (2004).

22 T. Vojta, Phys. Rev. Lett. 90, 107202 (2003).

23 T. Vojta, Phys. Rev. E 70, 026108 (2004).

24 T. Vojta, J. Phys. A 39, R143 (2006).

25 B. Fendler, R. Sknepnek, and T. Vojta, J. Phys. A 38, 2349 (2005).

${ }^{26}$ P. C. Hohenberg and B. I. Halperin, Rev. Mod. Phys. 49, 435 (1977).

27 N. Metropolis, A. Rosenbluth, M. Rosenbluth, and A. Teller, J. Chem. Phys. 21, 1087 (1953).

28 R. J. Glauber, J. Math. Phys. 4, 294 (1963).

29 I. M. Lifshitz, Usp. Fiz. Nauk 83, 617 (1964), [Sov. Phys.Usp. 7, 549 (1965)].

30 M. N. Barber, in Phase Transitions and Critical Phenomena, edited by C. Domb and J. L. Lebowitz (Academic, New York, 1983), vol. 8, p. 145.

31 U. Wolff, Phys. Rev. Lett. 62, 361 (1989).

32 S. Wansleben and D. P. Landau, Phys. Rev. B 43, 6006 
(1991).

33 P. Grassberger, Physica A 214, 547 (1995).

34 A. Jaster, J. Mainville, L. Schülke, and B. Zheng, J. Phys. A 32, 1395 (1999).
35 A. M. Ferrenberg and D. P. Landau, Phys. Rev. B 44, 5081 (1991).

36 M. Dickison and T. Vojta, J. Phys. A 38, 1199 (2005). 